\title{
Binary Operators and Connective Rules
}

\author{
V. CUTELLO \\ Department of Mathematics \\ University of Catania \\ Catania, Italy \\ E. MOLINA and J. MONTERO \\ Department of Statistics and O.R. \\ Complutense University \\ Madrid, Spain
}

\begin{abstract}
The main aim of this paper is to point out that a connective rule should be understood as a consistent family of connectives, in such a way that given any finite sequence of values we can evaluate its connective value. A connective rule is what we really need in practice, not a single connective operator. Only in some few cases we can characterize such a connective rule by means of a unique (associative) binary connective operator.
\end{abstract}

Key words: Associativity, Fuzzy Connectives.

\section{INTRODUCTION.}

It has been already acknowledged in the fuzzy literature that we apply different conjuntion and disjunction connectives (or different negation operations) depending on the context. Each decision maker may think a particular t-norm ( $t$-conorm) more appropriate than another $\mathbf{t}$-norm ( $\mathbf{t}$-conorm) depending on the problem he/she is being faced to. In general, we have to choose what we think is an appropriate amalgamation of the different pieces of information we have at each moment. For example, it was al- ready pointed out in Montero $[9,10]$ that at least we should be able to distinguish between the amalgamation of properties within the same charateristic, the amalgamation of properties belonging to different characteristics, and the amalgamation of objects. Each one of these amalgamation problems may be requiring different binary connectives, in such a way that all three should be simmultaneously included even in quite simple problems. Being different in nature, each one of these three amalgamation problem$s$ was solved by means of a unique associative and commutative binary connective. If we find the need of three amalgamation problems, then we look for three associative and commutative binary connectives. Once our binary amalgamation problem has been assumed associative, the final aggregated value can be obtained by successively applying such a binary operator to the given sequence of values in a one by one process, no matter the number of items under consideration. If in addition this binary operator is commutative, then such a successive calculus does not depend upon any particular order of the items.

Obviously, such a mathematical approach to connectiveness problems, by means of imposing associativity and commutativity, is an oversimplification of reality. Anyway, since we frequently do not know in 
advance the dimension of the input information, we still need a model capable of dealing with information of any arbitrary dimension. A connective rule should always be understood as a family of connective operators consistently solving any aggregation problem, no matter the number of items introduced as input. Only such a family of connective operators can be properly called connective rule. For example, as pointed out by in [1], an OWA operator [11], not being associative, does not define any $O W A$ rule.

Of course, not every family of binary connectives will actually be defining a connective rule. In this paper we consider the ability of a recursive calculus in order to model the desired consistency for connective rules, and this idea is applied to the OWA case in order to characterize consistent OWA rules.

\section{Connective Rules.}

Standard fuzzy connective operators for conjunction and disjunction ( $\mathrm{t}$-norms and $\mathrm{t}$-conorms; see, e.g., [8]) are assumed to be associative and commutative binary connectives. Hence, given any finite set of values in the unit interval, we can always evaluate its conjunction and its disjunction no matter the particular order in which those values are taken. We just succesively apply the same binary connective to the previous amalgamated value and a new value. A complete connective rule is therefore characterized by means of a unique binary connective aggregation (see also [6]).

Key fuzzy connectives are not associative. OWA (Ordered Weighted Averaging) operators, for example, are not associative. They were introduced by Yager [11] in order to fill the gap between min (which is the maximal t-norm) and $\max$ (which is the minimal t-conorm), on the basis of a previous re-arrangement of data according to the natural ordering.

DEFINITION 1 An OWA operator of dimension $n$ is a connective operator

$$
\phi:[0,1]^{n} \rightarrow[0,1]
$$

such that for any list $\left(a_{1}, \ldots, a_{n}\right)$ then

$$
\phi\left(a_{1}, \ldots, a_{n}\right)=\sum_{i=1}^{n} w_{i} a_{[i]} .
$$

for some associated list of weights $W=\left(w_{1}, \ldots, w_{n}\right)$ such that

1. $w_{i} \geq 0$ for all $i \in\{1, \ldots, n\}$

2. $\sum_{i=1}^{n} w_{i}=1$

The mean operator of $n$ numbers, for example, appears as the OWA operator of dimension $n$ with equal weights: $\left(w_{i}=1 / n\right.$ for all $\left.i=1, \ldots, n\right)$ :

$$
M_{n}:[0,1]^{n} \rightarrow[0,1]
$$

such that

$$
M_{n}\left(a_{1}, \ldots, a_{n}\right)=\frac{\sum_{i=1}^{n} a_{i}}{n} .
$$

Such a mean $M_{n}$ is just a single operator and it does not define the mean rule. When we refer to the mean rule, we mean the family of operators

$$
\left\{M_{n}\right\}_{n=2}^{\infty} \text {. }
$$

The mean rule is not a single mapping, but the above sequence of mappings. Rules should tell us in advance what we should be doing for any dimension of the information we get. Obviously, $M_{2}$ is not associative, but the mean rule

$$
\left\{M_{n}\right\}_{n=2}^{\infty}
$$

tells us how to evaluate the mean of any arbitrary finite set of numbers. The mean rule can be applied to any finite family of real numbers (moreover, without taking into account the particular ordering in which they have been arranged). In particular, any mean $M_{n}$ can be left and right recursively obtained from the previous means $M_{2}, \ldots, M_{n-1}$, where

$$
M_{2}(a, b)=(a+b) / 2
$$

and then

$$
\begin{array}{r}
M_{n}\left(a_{1}, \ldots, a_{n}\right)= \\
=\frac{(n-1) M_{n-1}\left(a_{1}, \ldots, a_{n-1}\right)+a_{n}}{n}= \\
=\frac{a_{1}+(n-1) M_{n-1}\left(a_{2}, \ldots, a_{n}\right)}{n}
\end{array}
$$


Several families of OWA operators have been introduced in the past (see, e.g., $[12,13,14]$ ). Being these families indeed interesting, they can not be properly understood as $O W A$ rules. A first proposal was given by Cutello-Montero [1] in order to formalize the notion of $O W A$ rule, on the basis of the existence of a recursive representation in terms of families of binary OWA operators applied to the re-arranged items (see also $[2,3,4]$ ). Such a representation enables OWA rules being operative, and capable of solving problems of arbitrary dimension.

In general, in order to allow left recursiveness, operators in a connective rule should allow for all $n$ the definition of a binary operator $L_{n}$ such that

$$
\begin{array}{r}
\phi_{n}\left(\pi\left(a_{1}\right), \ldots, \pi\left(a_{n-1}\right), \pi\left(a_{n}\right)\right)= \\
=L_{n}\left(\phi_{n-1}\left(\pi\left(a_{1}\right), \ldots, \pi\left(a_{n-1}\right)\right), \pi\left(a_{n}\right)\right) .
\end{array}
$$

Analogously, right recursiveness holds if and only if we can define a sequence $\left\{R_{n}\right\}_{n>1}$ such that

$$
\begin{array}{r}
\phi_{n}\left(\pi\left(a_{1}\right), \pi\left(a_{2}\right), \ldots, \pi\left(a_{n}\right)\right)= \\
=R_{n}\left(\pi\left(a_{1}\right), \phi_{n-1}\left(\pi\left(a_{2}\right), \ldots, \pi\left(a_{n}\right)\right)\right) .
\end{array}
$$

An interesting case to be analyzed will be that one in which both left and right recursions can share the same underlying ordering rule. That is, when

$$
\begin{array}{r}
\phi_{n}\left(a_{1}, \ldots, a_{n}\right)= \\
=R_{n}\left(\pi\left(a_{1}\right), \ldots, R_{2}\left(\pi\left(a_{n-1}\right), \pi\left(a_{n}\right)\right) \ldots\right) \\
=L_{n}\left(\ldots L_{2}\left(\pi\left(a_{1}\right), \pi\left(a_{2}\right)\right) \ldots, \pi\left(a_{n}\right)\right) .
\end{array}
$$

holds for some ordering rule $\pi$.

DEFINITION 2 If both left and right recursiveness hold for the same ordering rule, we then talk about it as a recursive rule.

In this way, recursiveness generalizes the concept of associativity, in the sense that recursive rules are the ones that can be evaluated iteratively (both sides), after an appropriate pre-arrangement of data. This ability of being iteratively evaluated was in fact the deep reason for associativity in practice (notice that the mean rule allows both left and right recursive definitions, although it is not associative).
In this paper we extend such an idea to more arbitrary connectives, in order to develope the concept of recursive connective rules [5].

\section{Final Comments.}

A connective rule is as a family of connectives that enables us to solve a variety of similar problems. Our approach is based upon recursiveness, but there are obviously other families of operators that represent rules in the sense that they allow the evaluation of any arbitrary number of items, not allowing the recursive approach as developed in this paper, but being consistent in some other alternative sense. This is the case, for example, of the Binomial OWA rule

$$
\left\{\phi_{n}\right\}_{n>1}
$$

where each $\phi_{n}$ is an OWA operator of dimension $n$ with weights

$$
w_{i, n}=\left(\begin{array}{c}
n \\
i
\end{array}\right) a^{i}(1-a)^{n-i} \quad \forall i=1, \ldots, n
$$

for some fixed $a \in(0,1)$. Each one of these operators can be recursively defined, but the family itself does not verify the recursive OWA rule condition given in definition 2. An operative description of such a family of OWA operators, still by means of a sequence of binary OWA operators and the natural decreasing ordering, can be developed by considering those families of OWA operators allowing a representation by means of the ordered linkage property (see [7]).

Acknowledgment: This research has been partially supported by Dirección General de Investigación Científica y Técnica (Spain).

\section{REFERENCES}

[1] V. Cutello and J. Montero. Recursive families of OWA operators. In: P.P. Bonissone, Ed., Proceedings of the Third IEEE Conference on Fuzzy Systems. IEEE Press, Piscataway (1994), 1137 1141.

[2] V. Cutello and J. Montero. Hierarchical aggregation of OWA operators: basic measures and related computational problems. Uncertainty, 
Fuzziness and Knowledge-Based Systems 3:1726 (1995).

[3] V. Cutello and J. Montero. The computational problems of using OWA operators. In: B. Bouchon-Meunier, R.R. Yager and L.A. Zadeh, Eds., Fuzzy Logic and Soft Computing. World Scientific, Singapore (1995)

[4] V. Cutello and J. Montero. Information and aggregation: ethical and computational issues. In: D. Ruan, Ed., Fuzzy Sets Theory and Advanced Mathematical Applications. Kluwer, Boston (1995)

[5] V. Cutello and J. Montero. Recursive connective rules. Technical Report.

[6] J. Dombi. Basic concepts for a theory of evaluation: the aggregative operator. European Journal of Operational Research, 10:282-293 (1982)

[7] J.C. Fodor, J.L. Marichal and M. Roubens. Characterization of the ordered weighted averaging operators. Institut de Mathemátique, $U$ niversité de Liège, Prépublication 93.011.

[8] G.J. Klir and T.A. Folger. Fuzzy sets, Uncertainty and Information. Prentice Hall, Englewood Cliffs, NJ (1988).

[9] J. Montero. Comprehensive fuzziness. Fuzzy Sets and Systems, 20:79-86 (1986)

[10] J. Montero. Extensive fuzziness. Fuzzy Sets and Systems, 21:201-209 (1987)

[11] R.R. Yager. On ordered weighted averaging aggregation operators in multi-criteria decision making. IEEE Transactions on Systems, Man and Cybernetics, 18:183-190 (1988)

[12] R.R. Yager. Families of OWA operators. Fuzzy Sets and Systems, 59:125-148 (1993)

[13] R.R. Yager. MAM and MOM operators for aggregation. Information Sciences, 69:259-273 (1993)
[14] R.R. Yager. Aggregation operators and fuzzy systems modeling. Fuzzy Sets and Systems, $67: 129-145$ (1994) 